\title{
A Comparative Study of Physiological Models on Cardiac Deformation Recovery: Effects of Biomechanical Constraints
}

\author{
Ken C.L. Wong ${ }^{1,2}$, Linwei Wang ${ }^{1}$, Huafeng Liu $^{3,1}$, and Pengcheng Shi ${ }^{1}$ \\ ${ }^{1}$ Computational Biomedicine Laboratory, Rochester Institute of Technology, Rochester, USA \\ \{linwei.wang, pengcheng.shi\}@rit.edu \\ ${ }^{2}$ ASCLEPIOS Research Project, INRIA Sophia Antipolis, Sophia Antipolis, France \\ chun-lok.wong@inria.fr \\ ${ }^{3}$ State Key Laboratory of Modern Optical Instrumentation, \\ Zhejiang University, Hangzhou, China \\ liuhf@zju.edu.cn
}

\begin{abstract}
Cardiac deformation recovery is to recover quantitative subject-specific myocardial deformation from imaging data. In the last decade, cardiac physiological models derived from anatomy, biomechanics, and cardiac electrophysiology have become increasingly popular in constraining the recovery problems because of their physiological meaningfulness. Although physiological models with various electrical and biomechanical components have been adopted by different frameworks and have exhibited promising results, these models have not been systematically compared under the same recovery framework, input data, and experimental setups. As different models comprise varying physiological plausibilities and complexities, comparisons under the same settings can aid choosing the proper models for specific goals and available resources. In this paper, under a state-space filtering framework for statistically optimal couplings between models and image data, we compare the performances of six different cardiac physiological models with different biomechanical constraints. Experiments were performed on synthetic data for quantitative comparisons, and on clinical data for their capabilities in identifying pathological situations.
\end{abstract}

\section{Introduction}

Cardiac deformation recovery is to recover quantitative subject-specific myocardial deformation from measurements. For noninvasive recoveries, medical images such as magnetic resonance images (MRI) provide an excellent source of in vivo anatomical and motion information. Nevertheless, as these measurements are sparse, projective, or noisy, and cannot directly provide quantitative cardiac deformation, a priori models are necessary to constrain the inverse problems for unique and meaningful results.

To provide physiologically meaningful constraints for deformation recovery from cardiac images, constraining models of different physiological plausibilities have started to be utilized in the last decade. In [1], a linear and isotropic biomechanical model (BM model) was used for statistical joint estimation of cardiac deformation and material properties. In [2], apart from the linear and transversely isotropic BM model with

G. Fichtinger, A. Martel, and T. Peters (Eds.): MICCAI 2011, Part I, LNCS 6891, pp. 420 427, 2011.

(c) Springer-Verlag Berlin Heidelberg 2011 


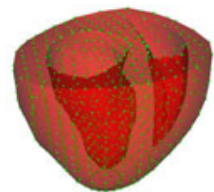

(a)

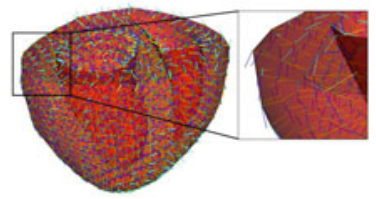

(b)

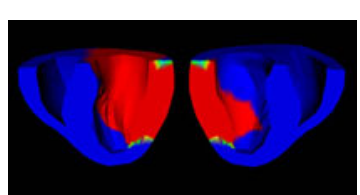

(c)

Fig. 1. Synthetic data. (a) Heart represented as nodes bounded by surfaces. (b) Tissue structure indicated by local coordinate systems ( $f, s, n$ : fiber, sheet, sheet normal: blue, yellow, cyan). (c) Infarcted regions shown in red.

fiber orientations considered, contraction forces were also simulated to provide an active deformation prior. In [3], a hyperelastic and orthotropic BM model was adopted with a nonlinear state-space filtering framework. The constraining models have become more physiologically plausible and complicated, from passive models with linear and isotropic materials to active models with hyperelastic and orthotropic materials.

These frameworks show promising results, however, as they were developed by different researchers at different times with different implementation difficulties, the models were not compared under the same recovery framework, input data, and experimental setups. As different models comprise varying physiological plausibilities and complexities, comparisons under the same settings can aid choosing the proper models for specific goals and available resources, so that unnecessary theoretical and implementation difficulties could be avoided. In view of this, we present here a comparative study of physiological models for cardiac deformation recovery from medical images using the recently developed framework in [3]. A nonlinearity-preserving state-space filtering framework based on unscented Kalman filter was used to couple physiological models with imaging data. Under this framework, the performances of six cardiac physiological models comprising different biomechanical material properties and force components were compared with the same input data and experimental settings. Experiments were performed on synthetic data for quantitative comparisons, and on clinical data for their capabilities in identifying pathological situations.

\section{Cardiac Physiological Models}

\subsection{Cardiac System Dynamics}

The heart is typically represented as a set of nodes bounded by the heart surfaces (Fig. 11a)). The matrix representation of the cardiac system dynamics is given as:

$$
\mathbf{M U ̈}+\mathbf{C} \dot{\mathbf{U}}+\mathbf{K} \Delta \mathbf{U}=\mathbf{F}
$$

where $\mathbf{M}, \mathbf{C}$, and $\mathbf{K}$ are the mass, damping, and stiffness matrices respectively. $\ddot{\mathbf{U}}$, $\dot{\mathbf{U}}$, and $\Delta \mathbf{U}$ comprise the respective nodal accelerations, velocities, and incremental displacements. F contains nodal external forces applied to the system. Different constraining models comprise different material properties in $\mathbf{K}$ and external forces in $\mathbf{F}$. 
Table 1. Physiological models compared in this study

\begin{tabular}{|l|c|c|c|c|c|c|}
\hline \hline \multirow{2}{*}{ Models } & \multicolumn{2}{|c|}{ Stress-strain relation } & \multicolumn{2}{|c|}{ Tissue structure } & \multicolumn{2}{|c|}{ External forces } \\
\cline { 2 - 7 } & Linear & Nonlinear & Isotropic & Anisotropic & Passive & Active \\
\hline LI-PBM & $\mathrm{X}$ & & $\mathrm{X}$ & & $\mathrm{X}$ & \\
\hline LI-APM & $\mathrm{X}$ & & $\mathrm{X}$ & & & $\mathrm{X}$ \\
\hline LTI-PBM & $\mathrm{X}$ & & & $\mathrm{X}$ & $\mathrm{X}$ & \\
\hline LTI-APM & $\mathrm{X}$ & & & $\mathrm{X}$ & & $\mathrm{X}$ \\
\hline HO-PBM & & $\mathrm{X}$ & & $\mathrm{X}$ & $\mathrm{X}$ & \\
\hline HO-APM & & $\mathrm{X}$ & & $\mathrm{X}$ & & $\mathrm{X}$ \\
\hline
\end{tabular}

\subsection{Physiological Models for Comparisons}

Physiological models with different material properties and external forces were tested. For the material properties in $\mathbf{K}$, hyperelastic and orthotropic (HO), linear and transversely isotropic (LTI), and linear and isotropic (LI) material models were tested. For the external forces in $\mathbf{F}$, the passive biomechanical model-based (PBM-based) recoveries with only image-derived forces, and the active physiological model-based (APMbased) recoveries with also simulated active contraction were tested. As the LI, LTI, or HO BM models can be used with either the PBM-based or the APM-based recoveries, a total of six models were studied (Table 1 ).

Material Properties. Different material properties associate with different assumptions of material nonlinearity and anisotropy of the myocardial tissue.

Hyperelastic and Orthotropic (HO) Material Model. Cardiac anatomy and biomechanics have shown that the myocardial tissue should be modeled as hyperelastic and orthotropic [4]. The HO model in [5] was used in the study, with strain energy function:

$$
\Psi(\boldsymbol{\epsilon})=\kappa(J \ln J-J+1)+\frac{1}{2} a_{\mathrm{BM}}\left(e^{Q}-1\right)
$$

where

$$
\begin{aligned}
Q= & b_{f f} \bar{\epsilon}_{f f}^{2}+b_{s s} \bar{\epsilon}_{s s}^{2}+b_{n n} \bar{\epsilon}_{n n}^{2} \\
& +b_{f s}\left(\bar{\epsilon}_{f s}^{2}+\bar{\epsilon}_{s f}^{2}\right)+b_{f n}\left(\bar{\epsilon}_{f n}^{2}+\bar{\epsilon}_{n f}^{2}\right)+b_{s n}\left(\bar{\epsilon}_{s n}^{2}+\bar{\epsilon}_{n s}^{2}\right)
\end{aligned}
$$

with $J$ the determinant of deformation gradient, and $\kappa$ the penalty factor for tissue incompressibility. $\bar{\epsilon}_{i j}$ are the isovolumetric components of the Green-Lagrange strain tensor $\epsilon . a_{\mathrm{BM}}$ and $b_{i j}$ are the material constants. The $f-s-n$ coordinate system represents the fibrous-sheet structure (Fig. 1 (b)).

Linear and Transversely Isotropic (LTI) Material Model. This model has been typically used for image-based cardiac deformation recovery [2]. The model is characterized by four material constants, $E_{f}, E_{c f}, \nu_{f}, \nu_{c f}$, which are the Young's moduli and Poisson's ratios along and across the fiber respectively.

Linear and Isotropic (LI) Material Model. This model was used in the earlier approaches [1]. The model is characterized by two material constants, with Young's modulus $E=$ $E_{f}=E_{c f}$ and Poisson's ratio $\nu=\nu_{f}=\nu_{c f}$. 
External Force Components. The force vector $\mathbf{F}$ in (1) drives the deformation, which can be provided by enforcing displacements of salient cardiac features derived from images [1], or by active contraction simulated using electrophysiological models [2]3].

Passive Biomechanical Model-Based(PBM-Based) Recoveries. The BM models are used without active contraction stresses. In consequence, the deformations are introduced passively by the image information through the filtering process in Section 3 .

Active Physiological Model-Based (APM-Based) Recoveries. The BM models are used with simulated active contraction stresses. A two-variable diffusion-reaction system has been adopted to model the action potential propagation [6], which is further transformed into active stresses through the electromechanical coupling model in [2].

\section{Cardiac Deformation Recovery with Statistically Optimal Model-Measurement Coupling}

The cardiac physiological models provide physiological constraints for cardiac deformation recovery. Nevertheless, as the models are not subject-specific, and the measurements extracted from images are not perfect, a nonlinear state-space filtering framework is required to couple the measurements with the models according to their own merits.

To utilize state-space filtering, the stochastic state-space equations:

$$
\begin{aligned}
& \mathbf{x}(k)=f(\mathbf{x}(k-1))+\boldsymbol{\omega}(k-1) \\
& \mathbf{y}(k)=h(\mathbf{x}(k))+\boldsymbol{\nu}(k)
\end{aligned}
$$

need to be defined. In (4), $\mathrm{x}$ is the state vector to be estimated with model uncertainties $\boldsymbol{\omega}$, and $f$ projects $\mathbf{x}$ from time step $k-1$ to $k$. In (5), $\mathbf{y}$ is the measurement vector with measurement errors $\boldsymbol{\nu}$, and $h$ relates the state to the measurements.

For cardiac deformation recovery, $\mathbf{x}$ is the nodal displacement vector $\mathbf{U}$. Using the relation $\mathbf{U}(k)=\mathbf{U}(k-1)+\Delta \mathbf{U}$, we have the concrete form of (4) as:

$$
\mathbf{U}(k)=f(\mathbf{U}(k-1))+\boldsymbol{\omega}(k-1)=\mathbf{U}(k-1)+\Delta \mathbf{U}+\boldsymbol{\omega}(k-1)
$$

where $\Delta \mathbf{U}$ is obtained by solving (1), in which $\mathbf{K}$ depends nonlinearly on $\mathbf{U}$.

The concrete form of (5) is given as:

$$
\mathbf{y}(k)=\mathbf{H x}(k)+\boldsymbol{\nu}(k)
$$

with $y$ the nodal displacements of salient cardiac features extracted from images through motion tracking, and $\mathbf{H}$ the measurement matrix relating $\mathbf{U}$ to $\mathbf{y}$.

With (45), the model and measurements are connected together, and state-space filtering can be performed to obtain the optimal estimation. To preserve model nonlinearity and maintain computational feasibility, we utilize the unscented Kalman filter which comprises the advantages of Monte Carlo methods and Kalman filter updates [7]. 


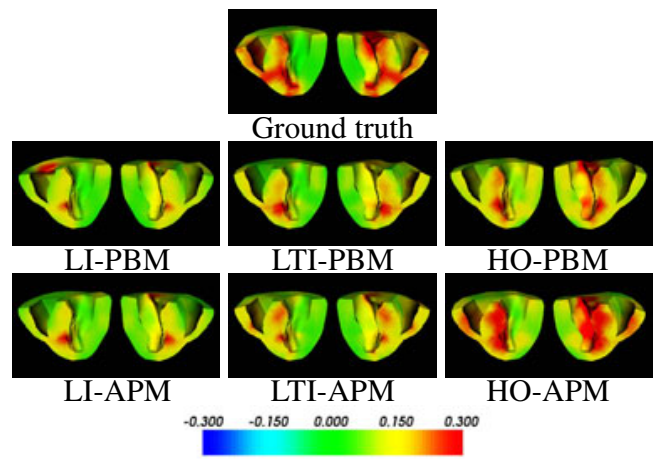

(a)

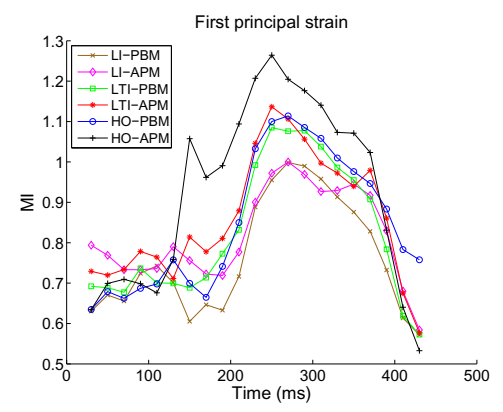

(b)

Fig. 2. Synthetic data. (a) First principal strains at the end of systole (250 ms). (b) Mutual information between strain patterns of the ground truth and the recovered deformations for the whole cardiac cycle.

\section{Comparisons of Physiological Models}

The model parameters were adopted from the literature. For the HO BM model, $a_{\mathrm{BM}}=$ $0.88 \mathrm{kPa}, b_{f f}=6, b_{s s}=7, b_{n n}=3, b_{f s}=12, b_{f n}=3, b_{s n}=3$, and $\kappa=5 \times 10^{4}$ [5]. For the LTI BM model, $E_{f}=75 \mathrm{kPa}, E_{c f}=25 \mathrm{kPa}, \nu_{f}=0.4$, and $\nu_{c f}=0.2$. For the LI BM model, $E=75 \mathrm{kPa}$ and $\nu=0.4$ [1].

\subsection{Synthetic Data}

Experimental Setups. The heart architecture from the University of Auckland was used to provide the cardiac geometry and tissue structure [8], and a heart representation of 1746 nodes was constructed (Fig. 1 (a) and (b)). To verify the capabilities of locating diseased areas, some regions of the heart were set to be infarcted (Fig.1(c)), where the electricity could not propagate through, and the stiffness was set to be three times as that in the normal regions. With this setting, the HO BM model in Section 2.2 was used to simulate a cardiac cycle of $450 \mathrm{~ms}$ as the ground truth. The nodal displacements on the heart boundaries were extracted and noises of $10 \mathrm{~dB}$ signal-to-noise ratio were added, which were treated as the measurement inputs from medical images.

Results and Discussion. Fig. 22 a) shows the first principal strain maps for visual comparisons, as there is a strong inverse relation between the first principal strain and the extent of myocardial infarction [9]. There is almost no deformation in the infarcted regions in the ground truth. This observation also appears in the recovered strains of different models, but different models possess different similarities to the ground truth. To quantify the similarities of patterns rather than the absolute values, mutual information comparison is used (Fig. 2(b)). Higher mutual information implies higher similarity.

The strain maps and mutual information show that the PBM-based and APM-based recoveries behave similarly, as the measurements on the heart surfaces provided the displacement boundary conditions of the deformations. When the boundary conditions of 

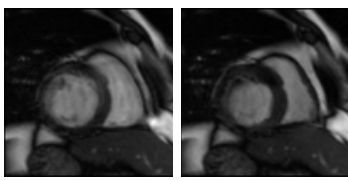

(a)

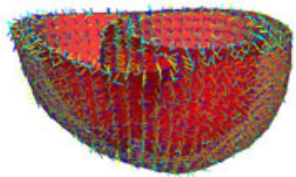

(b)

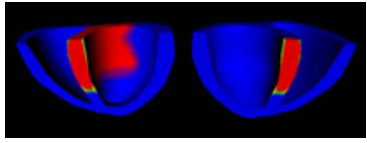

(c)

Fig. 3. Patient data. (a) MRI at end of diastole and end of systole. (b) Cardiac geometry segmented from the first frame of the MRI sequence, with mapped tissue structure $(f, s, n$ : blue, yellow, cyan). (c) Infarcted segments $(3,4,9,10)$ shown in red.

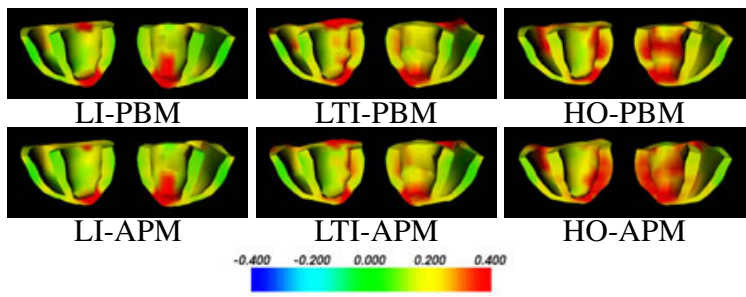

Fig. 4. Patient data. First principal strains at the end of systole.

most surfaces were given, the effects of the active stresses decreased. Such similarities are more obvious for the LI and LTI models, as the active forces were calibrated using the HO-APM model and only caused relatively small deformations when applied to the linear BM models. Therefore, the active forces did not contribute much to the recovery.

On the other hand, the differences between different BM models are more obvious. The differences between the LI and the LTI models are very clear, especially around the end of systole. As the only differences between these two linear BM models are the Young's modulus $E_{c f}$ and the Poisson's ratio $\nu_{c f}$, this shows the importance of using anisotropic BM model. For the HO models, the HO-APM model has the highest MI, but the HO-PBM model behaves very similar to the LTI-models. Therefore, HO models need to be used with proper active contraction stresses to gain the benefits.

\subsection{Patient Data}

Experimental Setups. The data sets are available in [10], for case 1 and case 2 from two patients with acute myocardial infarction, with the infarcted regions identified by experts. Case 1 contains a human short-axis MRI sequence of 19 frames in one cardiac cycle (52.5 ms/frame), with 12 slices/frame, $8 \mathrm{~mm}$ inter-slice spacing, and in-plane resolution $1.32 \mathrm{~mm} /$ pixel. Case 2 contains a human short-axis MRI sequence of 16 frames in one cardiac cycle (50 ms/frame), with 13 slices/frame, $8 \mathrm{~mm}$ inter-slice spacing, and in-plane resolution $1.32 \mathrm{~mm} /$ pixel. In this paper, only the figures of case 2 are shown for illustration. Fig. 3(a) shows the MRI. Segmentations were performed to obtain the heart geometries at the end of diastole, and the fibrous-sheet structures were mapped from the Auckland heart architecture using nonrigid registration (Fig. 3 b)). Fig. 3 (c) shows the infarcted regions identified by experts from contrast-enhanced MRI with the 
Table 2. Patient data. Segment numbers at different levels sorted by the mean of the first principal strains of each segment at the end of systole, in ascending order. The numbers of the infarcted segments are highlighted in red. The optimal cutoff segments for identifying infarcted segments are indicated through double vertical lines $(\|)$.

\begin{tabular}{|c|cccc|cc|ccc||ccc|ccc|c|}
\hline \hline Case 1 & \multicolumn{1}{|c|}{ Basal } & \multicolumn{10}{|c|}{ Mid } & \multicolumn{3}{|c|}{ Apical } \\
\hline LI-PBM & 3 & 2 & 4 & 1 & 6 & 5 & 10 & 9 & 8 & 7 & 11 & 12 & 14 & 15 & 13 & 16 \\
\hline LI-APM & 3 & 4 & 2 & 1 & 6 & 5 & 10 & 9 & 8 & 11 & 7 & 12 & 14 & 15 & 13 & 16 \\
\hline LTI-PBM & 3 & 4 & 5 & 2 & 1 & 6 & 9 & 10 & 8 & 11 & 7 & 12 & 14 & 13 & 15 & 16 \\
\hline LTI-APM & 3 & 4 & 2 & 5 & 1 & 6 & 9 & 8 & 10 & 11 & 7 & 12 & 14 & 13 & 15 & 16 \\
\hline HO-PBM & 3 & 2 & 4 & 1 & 5 & 6 & 9 & 8 & 10 & 12 & 7 & 11 & 14 & 13 & 15 & 16 \\
\hline HO-APM & 3 & 2 & 4 & 1 & 5 & 6 & 8 & 9 & 10 & 12 & 7 & 11 & 14 & 13 & 15 & 16 \\
\hline
\end{tabular}

\begin{tabular}{|c|c|c|c|c|c|}
\hline$\overline{\mathrm{Ca}}$ & & Basal & & Mid & $\overline{\mathrm{Ap}}$ \\
\hline LI-PBM & & $\begin{array}{|lllll|}6 & 5 & 1 & 2 & 4 \\
\end{array}$ & $\begin{array}{ll}9 & 12 \\
\end{array}$ & \begin{tabular}{l||lll}
10 & 8 & 7 & 11 \\
\end{tabular} & $\begin{array}{lllll}15 & 14 & 16 & 13 \\
\end{array}$ \\
\hline LI-APM & & $\begin{array}{|llllll|}6 & 5 & 1 & 4 & 2 \\
\end{array}$ & $9 \quad 12$ & $\begin{array}{lll}8 & 11 & 7\end{array}$ & $\begin{array}{llll}15 & 14 & 16 & 13\end{array}$ \\
\hline $11-1$ & & $\begin{array}{|lllll|}2 & 1 & 6 & 5 & 4 \\
\end{array}$ & \begin{tabular}{ll||}
9 & 10 \\
\end{tabular} & $\begin{array}{ll}8 & 11 \\
\end{array}$ & $\begin{array}{llll}15 & 14 & 16 & 13 \\
\end{array}$ \\
\hline LTI-APM & & $\begin{array}{|lllll|}2 & 5 & 1 & 6 & 4 \\
\end{array}$ & \begin{tabular}{ll||}
9 & 10 \\
\end{tabular} & \begin{tabular}{|llll}
12 & 8 & 7 & 11 \\
\end{tabular} & $\begin{array}{llll}15 & 14 & 16 & 13\end{array}$ \\
\hline HO-PBM & & 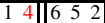 & 109 & \begin{tabular}{|lll}
11 & 12 & 8
\end{tabular} & \begin{tabular}{|lllll}
15 & 14 & 16 & 13
\end{tabular} \\
\hline & & \begin{tabular}{ll||lll}
3 & 4 & 5 & 6 & 2
\end{tabular} & & $\begin{array}{lll}7 & 11 & 8\end{array}$ & $\begin{array}{llll}15 & 14 & 16 & 13\end{array}$ \\
\hline
\end{tabular}

Table 3. Patient data. Optimal sensitivities (SEN) and specificities (SPEC) for identifying infarcted segments.

\begin{tabular}{|c|c|c|c|}
\hline \hline \multirow{2}{*}{ Case 1 } & Basal & Mid & Apical \\
\cline { 2 - 4 } & SEN / SPEC & SEN / SPEC & SEN / SPEC \\
\hline LI-PBM & $100 \% / 67 \%$ & $100 \% / 75 \%$ & $100 \% / 100 \%$ \\
\hline LI-APM & $100 \% / 67 \%$ & $100 \% / 75 \%$ & $100 \% / 100 \%$ \\
\hline LTI-PBM & $100 \% / 33 \%$ & $100 \% / 75 \%$ & $100 \% / 100 \%$ \\
\hline LTI-APM & $67 \% / 67 \%$ & $100 \% / 100 \%$ & $100 \% / 100 \%$ \\
\hline HO-PBM & $100 \% / 67 \%$ & $100 \% / 100 \%$ & $100 \% / 100 \%$ \\
\hline HO-APM & $100 \% / 67 \%$ & $100 \% / 100 \%$ & $100 \% / 100 \%$ \\
\hline
\end{tabular}

\begin{tabular}{|c|c|c|}
\hline \hline \multirow{2}{*}{ Case 2} & Basal & Mid \\
\cline { 2 - 3 } & SEN / SPEC & SEN/ SPEC \\
\hline LI-PBM & $50 \% / 100 \%$ & $100 \% / 75 \%$ \\
\hline LI-APM & $50 \% / 100 \%$ & $100 \% / 75 \%$ \\
\hline LTI-PBM & $50 \% / 100 \%$ & $100 \% / 100 \%$ \\
\hline LTI-APM & $50 \% / 100 \%$ & $100 \% / 100 \%$ \\
\hline HO-PBM & $100 \% / 75 \%$ & $100 \% / 100 \%$ \\
\hline HO-APM & $100 \% / 75 \%$ & $100 \% / 100 \%$ \\
\hline
\end{tabular}

segmentation and nomenclature suggested by the American Heart Association. The infarcted segments of case 1 are 1, 2, 3, 8, 9, 13, 14, 15, and of case 2 are 3, 4, 9, 10 . As short-axis MRI cannot provide accurate motion at the apex, segment 17 was not considered in our experiments. To obtain the measurement inputs, a registration framework based on free-form deformation was used to extract the displacement field from the image sequences [11], and the displacements on the current heart boundaries were utilized. The infarcted regions were unknown in the recoveries.

Results and Discussion. The qualitative assessments are shown in Fig. 4, The strain patterns of all models show relatively small deformations in the infarcted regions. Similar to the synthetic data, the PBM-based and the APM-based models are relatively similar, and the differences between different BM models are more obvious. All explanations for the strain patterns of the synthetic data are applicable here.

For clinical applications, the capabilities of frameworks in locating diseased regions are of great interest, thus the results are compared in this aspect. We sorted the segments at different levels by the mean of the first principal strains of each segment at the end of systole, in ascending order (Table 2). From Table 2, we can calculate the various sensitivities and specificities of the frameworks with different cutoff segments, where sensitivities are the ratios between the correctly identified positives (infarcted segments) and the actual positives, and the specificities are the ratios between the correctly identified negatives (normal segments) and the actual negatives. The optimal sensitivities and specificities are shown in Table 3. As there is no infarction at the apical level for case 2 , the corresponding optimal sensitivities and specificities are unavailable.

Table 2 shows again that the differences between PBM-based and APM-based models are small. Table 3 shows that the HO models have the best capabilities in both case 1 and 2. Comparing between the LTI and LI models, in case 1, the LTI models have 
better capabilities than the LI models at the mid-ventricular level but worse capabilities at the basal level. In case 2, the LTI models have better capabilities at the mid-ventricular level than the LI models. Considering also the results in the synthetic experiments, the LTI models perform better than the LI models.

\section{Conclusion}

The results show that when image information is used, the active contraction stresses do not contribute much in the recovery. Therefore, if subject-specific active contraction stresses are unavailable but the image quality is good, the PBM-based models can be the proper choices. On the other hand, the anisotropies of the BM models have more impacts on the results, but the HO models do not necessarily provide much better results than the LTI models especially in verifying infarcted regions. Therefore, if the absolute strain values are unimportant, the use of LTI models might be enough.

\section{References}

1. Shi, P., Liu, H.: Stochastic finite element framework for simultaneous estimation of cardiac kinematic functions and material parameters. Medical Image Analysis 7, 445-464 (2003)

2. Sermesant, M., Delingette, H., Ayache, N.: An electromechanical model of the heart for image analysis and simulation. IEEE Transactions on Medical Imaging 25(5), 612-625 (2006)

3. Wong, K.C.L., Wang, L., Zhang, H., Shi, P.: Nonlinear cardiac deformation recovery from medical images. In: IEEE International Conference on Image Processing, pp. 2513-2516 (2009)

4. Glass, L., Hunter, P., McCulloch, A. (eds.): Theory of Heart: Biomechanics, Biophysics, and Nonlinear Dynamics of Cardiac Function. Springer, Heidelberg (1991)

5. Usyk, T.P., Mazhari, R., McCulloch, A.D.: Effect of laminar orthotropic myofiber architecture on regional stress and strain in the canine left ventricle. Journal of Elasticity 61, 143-164 (2000)

6. Aliev, R.R., Panfilov, A.V.: A simple two-variable model of cardiac excitation. Chaos, Solitons \& Fractals 7(3), 293-301 (1996)

7. Julier, S.J., Uhlmann, J.K.: Unscented filtering and nonlinear estimation. Proceedings of the IEEE 92(3), 401-422 (2004)

8. Nash, M.: Mechanics and Material Properties of the Heart using an Anatomically Accurate Mathematical Model. PhD thesis, The University of Auckland (1998)

9. Tim Marcus, J., Götte, M.J.W., van Rossum, A.C., Kuijer, J.P.A., Heethaar, R.M., Axel, L., Visser, C.A.: Myocardial function in infarcted and remote regions early after infarction in man: assessment by magnetic resonance tagging and strain analysis. Magnetic Resonance in Medicine 38(5), 803-810 (1997)

10. PhysioNet/Computers in Cardiology challenge: electrocardiographic imaging of myocardial infarction (2007), http: / / www . physionet .org/challenge/2007/

11. Perperidis, D., Mohiaddin, R.H., Rueckert, D.: Spatio-temporal free-form registration of cardiac MR image sequences. Medical Image Analysis 9(5), 441-456 (2005) 